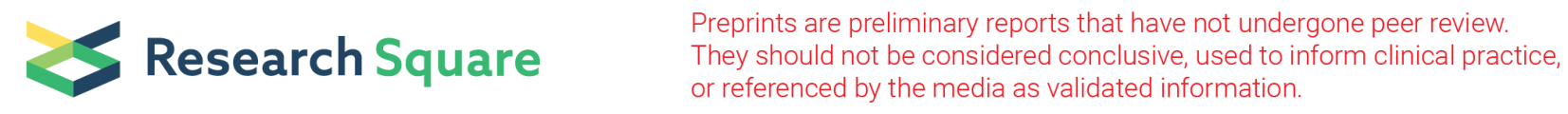

\title{
Longitudinal prospective evaluation of oral health-related quality of life in young patients undergoing fixed orthodontic appliance treatment as part of a randomised trial
}

Mustafa Elhussein ( $\nabla$ mustafa_elhussein@hotmail.com )

University of Sheffield https://orcid.org/0000-0003-1353-5840

Philip Benson

University of Sheffield

\section{Research article}

Keywords: Patient-reported outcomes, Oral health related quality of life, PIDAQ, Malocclusion, Orthodontic treatment

Posted Date: May 17th, 2020

DOI: https://doi.org/10.21203/rs.3.rs-25214/v1

License: (c) (i) This work is licensed under a Creative Commons Attribution 4.0 International License. Read Full License 


\section{Abstract \\ Background}

The objectives of this study were to investigate relationships between change in the aesthetic appearance before and after orthodontic treatment and patientreported change in oral health-related quality of life (OHRQoL), and to assess the responsiveness of two OHRQoL measures to any changes from orthodontic treatment.

\section{Methods}

Two hundred and ten participants in a multicentre (two teaching hospitals and four specialist orthodontic practices), single blinded, randomised clinical trial with 2 parallel groups, were administered one of two age-specific questionnaires, either the Child Perceptions Questionnaire (CPQ11-14-ISF-16) or the Psychosocial Impact of Dental Aesthetics Questionnaire (PIDAQ), before and after orthodontic treatment. Clinical photographs were assessed by two groups of laypeople and orthodontists using the Index of Orthodontic Treatment Need Aesthetic Component (IOTN-AC).

\section{Results}

Two hundred and ten participants were randomised, and 197 completed the trial. Before and after OHRQoL data were successfully obtained from 110 participants. There was a mean reduction in the total CPQ11-14-ISF-16 scores of $3.9(\mathrm{SD}=8.0)$, and a mean reduction of $34.2(\mathrm{SD}=18.5)$ in the total PIDAQ score. CPQ11-14-ISF-16 demonstrated poor longitudinal construct validity $(p=0.155)$. Correlations between the change in total CPQ11-14-ISF-16 scores and change in IOTN-AC assessments were small for both orthodontists $(r=-0.084 ; p=0.516)$ and laypeople $(r=-0.140 ; p=0.225)$. There were higher associations between the improvement in the Social well-being (SWB) subdomain and improvement in IOTN-AC. Correlations between the changes in total PIDAQ scores and the IOTN-AC assessments, were higher in comparison to the correlations with CPQ11-14-ISF-16 (orthodontists; $r=0.223$, laypeople; $r=0.025$ ). There were no adverse effects.

\section{Conclusion}

CPQ11-14-ISF-16 and IOTN-AC measure different attributes. This demonstrated the role of SWB on children OHRQoL. Condition-specific measures (PIDAQ) are more responsive to change in self-reported OHRQoL than generic measures (CPQ11-14-ISF-16) after treatment.

\section{Trial Registration:}

The trial was registered at ClinicalTrials.gov NCT01925924.

\section{Background}

The Index of Orthodontic Treatment Need Aesthetic Component (IOTN-AC) aims to objectively assess the level of normative need for orthodontic treatment; ${ }^{1}$ however it fails to consider the patient and/or parental opinions and perspectives. Studies conducted within different populations and health systems have found that the demand for orthodontic treatment is multifactorial and mostly determined by cultural, ethnic and socio-economic factors. ${ }^{2}$ Supplementing clinical indicators of normative need, such as the Index of Orthodontic Treatment Need Aesthetic Component, with patient-reported measures, might bridge the gap between the differences in the opinion of patients' and orthodontists' perception towards orthodontic treatment need and provision.

The concept of oral health-related quality of life (OHRQoL) has been developed to describe the impact of oral disease and conditions on the everyday lives of individuals. Sischo et al., ${ }^{3}$ define oral health-related quality of life as: "a multidimensional construct that includes a subjective evaluation of the individual's oral health, functional well-being, emotional well-being, expectations and satisfaction with care, and sense of self." There is evidence that individuals with malocclusion have a worse oral health-related quality of life as ${ }^{4}$ and that treatment of malocclusion improves oral health-related quality of life as in young people. ${ }^{5}$

Several generic oral health-related quality of life measures have been developed and validated; however some investigators have questioned whether a generic measure, designed to compare the impacts of a range of oral diseases and conditions on OHRQoL, can capture all the impacts of specific conditions, such as malocclusion. ${ }^{6}$ It has been suggested that condition-specific measures might be more appropriate for assessing the impacts of some conditions and for measuring any changes after treatment. ${ }^{7}$

One condition-specific measure is the Psychosocial Impact of Dental Aesthetics Questionnaire (PIDAQ), which was developed, in young adults (aged 1830 years) to assess the orthodontic specific aspects of OHRQoL. ${ }^{8}$ The Psychosocial Impact of Dental Aesthetics Questionnaire has been validated crossculturally 9,10 and has been found to have some correlation with occlusal indices, such as the Dental Aesthetic Index. ${ }^{11}$ Furthermore, the Psychosocial Impact of Dental Aesthetics Questionnaire has been tested in a large sample of 1112 children and young adults in Germany (aged 11-17 years) and the questionnaire demonstrated good psychometric properties and validity for use among adolescents. ${ }^{12}$

The objectives of this study were: 
- To investigate relationships between change in the aesthetic appearance before and after orthodontic treatment and the patient-reported change in OHRQoL;

- To assess the responsiveness of two OHRQoL measures to any changes from orthodontic treatment.

We hypothesized that there is a positive correlation between the change in dental aesthetics after orthodontic treatment and patient-reported change in OHRQoL.

\section{Methods \\ Ethics}

Ethical approval was obtained from the National Research Ethics Service Committee of Yorkshire and the Humber (Leeds West) Ethics Research Committee (reference number 07/H1307/ 153; October 24, 2007) in the United Kingdom and the Clinical Research Ethics Committee of Cork Teaching Hospitals (reference number ECM5(2)5208; February 6, 2008) in the Republic of Ireland. Also, ethical approval was obtained from the Ethics Research Committee of the School of Clinical Dentistry (Ref:013575-19/05/2017). Written informed consent was obtained from all participants and their parents or guardians, but participants were free to withdraw consent at any stage. The trial was registered in a clinical trials registration database (ClinicalTrials.gov; NCT01925924). There were no changes to the protocol after trial commencement.

\section{Design, participants, eligibility criteria, and setting}

In this study, we used a before and after longitudinal design to assess the changes in OHRQoL after orthodontic treatment. The longitudinal data were obtained before and 3 months after orthodontic treatment from participants recruited to a multi-center, single-blinded, randomised controlled clinical trial with 2 parallel groups, involving two teaching hospitals and four specialist orthodontic practices. Initially, eight centers participated in the study (six specialist practices and two teaching hospitals); however, 1 center recruited only 2 participants and 1 recruited 7; hence, data collection from these centers was incomplete. Subsequently, they were withdrawn from the study. To minimize performance bias, 6 specialist orthodontists treated the participants for the entire duration of treatment.

Participants were asked to complete one of two OHRQoL questionnaires. Participants aged 11 to 14 years were asked to complete the Short Form Child Perceptions Questionnaire (CPQ11-14-ISF-16) before the placement of their orthodontic fixed appliance and 3 months after removal. Participants aged 15 years and older were asked to complete PIDAQ at the same timepoints.

Digital colour intra-oral frontal images of the participants' teeth before and after treatment were assessed by 11 orthodontists (7 females and 4 males) and 16 laypeople (9 females and 7 males) using IOTN-AC (Fig. 1).

\section{Inclusion/exclusion criteria}

We recruited patients that were about to commence fixed appliance orthodontic treatment. The inclusion criteria were (1) 11 years of age or older, in good general health; (2) oral hygiene considered by the clinician to be adequate for fixed orthodontic appliances; and (3) require upper and/or lower fixed orthodontic appliance treatment.

We excluded patients with cleft lip and palate and patients who required multidisciplinary orthodontic and orthognathic surgery.

\section{Outcomes and any changes after trial commencement}

The details of the primary outcomes including how and when they were assessed have been previously reported and published. ${ }^{13}$ The primary outcomes were the presence or absence of new demineralized lesions (DLs), evaluated using the clinical photographic images obtained from before and after orthodontic treatment, and judgments about the esthetic appearance of any new demineralized lesions and the number of first-time bond failures during treatment, taken from the clinical record.

The secondary outcomes were the relationships between the change in aesthetic appearance before and after orthodontic treatment and patient-reported change in OHRQoL, and the responsiveness of two OHRQoL measures to any changes from orthodontic treatment. There were no changes to the outcomes after trial commencement.

\section{Online Survey}

IOTN-AC assessments were made via an online survey designed specifically for the project and developed by Oasis Computer Systems@ . The website was developed using a combination of JavaScript and ASP.NET framework (Fig. 1). The Lay people were non-dental students, who were approached at the university campus and then received an electronic information pack and link to the survey by e-mail. The clinicians were members of the orthodontic consultant group, who were invited to take part by e-mail and then received an electronic pack. The survey started with some information about the study, and the assessors were then informed that by continuing with the survey they would be considered to have given their consent to take part. The assessors were asked to rate each photograph on a scale of 1 to 10 using the validated IOTN-AC, ${ }^{1}$ which consists of ten photographs of the front teeth showing a range of malocclusions from 1 (most attractive or straight teeth) to 10 (least attractive or crooked teeth).

\section{Sample size calculation}


The sample size for the trial was based on the primary outcome, which was the difference in the prevalence of demineralisation between those participants bonded with resin modified glass ionomer cement (RM-GIC) and those bonded with composite. It was calculated according to the method of Altman for comparing the proportions of binary data. It was determined that a sample size of 200 patients (100 in each group) would be required to detect a $20 \%$ reduction in the prevalence of new DLs between those bonded with RM-GIC and those bonded with LCC (significance level of 0.05 and power of 0.85 ). To account for an estimated potential withdrawal or dropout rate of $20 \%$, a total of 240 participants would need to be recruited.

\section{Interim analyses and stopping guidelines}

No interim analyses were planned. No adverse events were encountered; hence the stopping guidelines were not introduced.

\section{Randomisation}

Randomisation was carried out using a computer generated random number sequence to produce a random sample stratified on the operator. This ensured that each operator was allocated the same number of patients in the 2 groups. Blocked randomisation was used to keep the 2 groups equal. Subjects were allocated using sequentially numbered opaque envelopes at each center.

\section{Blinding}

The trial was single-blinded, because it was not possible to mask either the clinician carrying out the treatment or the participants to the type of bonding adhesive used; however, the examiners assessing the clinical photographic images were masked to group allocation.

\section{Statistical Analysis (secondary outcomes)}

Details of the statistical methods and additional analyses used to compare groups for primary outcomes were previously published. ${ }^{13}$ CPQ11-14-ISF-16 consists of three global questions, followed by 16 specific impact questions in four subdomains. The response to the global questions was given using a 5 point response format ranging from 'Excellent' $=0$ to 'Poor' $=4$ and from 'Not at all' $=0$ to 'Very much'. The responses to the impact questions are in the form of a five-part scale recording the frequency of the impact (from 'Never' $=0$ to 'Everyday or almost everyday'=4). The responses from each impact question were added together to obtain a total score. A high score indicated poorer OHRQoL. CPQ11-14-ISF-16 consisted of 4 subdomains that measures aspects of OHRQoL in children: Oral Symptoms (OS), Functional Limitation (FL), Emotional Well-being (EWB), and Social Well-being (SWB). ${ }^{14}$ The questionnaire was developed and validated in Canada in 2002. Since then it has been validated globally for use in children with malocclusion. ${ }^{15}$

PIDAQ consists of 23 statements (e.g. 'I am proud of my teeth'; 'I like to show my teeth when I smile') and responses are on a 5-point scale from 0 'Not at all' to 4 'Very Much'. Unlike CPQ11-14-ISF-16, PIDAQ consists of both negative and positive impacts. The scoring of the positive questions was reversed, so the higher score always represented worse OHRQoL. The responses of all 23 items were summed to obtain an overall score.

The means, SD and 95\% confidence intervals of the total and subdomain CPQ11-14-ISF-16 and PIDAQ scores were calculated and the distribution of differences between the before and after treatment scores were examined for normality. To evaluate the responsiveness of CPQ11-14-ISF-16 and PIDAQ to change in OHRQoL following orthodontic treatment effect sizes were calculated using the following formula:

\section{$\underline{\text { Before score - after score }}$}

\section{SD of differences}

Pearson Product correlation coefficients were calculated to determine any associations between change in the aesthetic appearances of the teeth and change in CPQ11-14-ISF-16 and PIDAQ total scores and subdomain scores. The obtained data were analyzed using the Statistical Package for Social Sciences (SPSS version 19, SPSS inc, Chicago, III) software, and the statistical significance was set at $\mathrm{p}<0.05$.

\section{Results}

Recruitment started in February 2009 and was completed in March 2012. The first patient completed treatment in September 2010 and the last by December 2014. Two hundred and ten patients were randomised, and the flow of participants through the study is shown in Fig. 2 . The results of the primary outcomes were previously reported and published. ${ }^{13}$

Before and after OHRQoL data were successfully obtained from 110 participants (CPQ11-14-ISF-16 = 68; PIDAQ = 42) (Fig. 2). The baseline demographic data are shown in Table 1. The descriptive data for the before treatment, 3 months after treatment and changes in the total and sub-domain CPQ11-14-ISF-16 scores and the PIDAQ total scores, as well as the effect sizes are shown in Table 2. The mean total CPQ11-14-ISF-16 score before treatment was 13.4 (SD = 7.6; $95 \% \mathrm{Cl} 11.7-15.2)$ and after treatment was 9.8 ( $\mathrm{SD}=7.4 ; 95 \% \mathrm{Cl} 8.1-11.6)$. The mean change in the total score was therefore $3.9(\mathrm{SD}=8 ; 95 \% \mathrm{Cl} 2-5.8)$. The effect size for the change in the total CPQ11-14-ISF-16 scores before and after treatment was "medium", according to the definitions of Cohen 16 (Table 2). 
Table 1

Baseline demographics for participants in Oral health-related quality of life analysis

\begin{tabular}{|c|c|c|c|c|}
\hline \multirow{4}{*}{ Male: female } & \multicolumn{4}{|c|}{ All participants $n=110$} \\
\hline & \multicolumn{4}{|l|}{$39: 71$} \\
\hline & \multirow[t]{2}{*}{ mean } & \multirow[t]{2}{*}{ SD } & \multicolumn{2}{|l|}{ range } \\
\hline & & & minimum & maximum \\
\hline Age (y) & 15.5 & 3.3 & 10.9 & 33.7 \\
\hline Average length of treatment (mo) & 17.6 & 7.1 & 3 & 43 \\
\hline
\end{tabular}

Table 2

Child Perceptions Questionnaire, and Psychosocial Impact of Dental Aesthetics Questionnaire total scores, subdomain scores, change scores and effect sizes from before and after treatment

\begin{tabular}{|c|c|c|c|c|c|c|c|c|c|c|c|c|c|c|c|c|}
\hline \multirow{3}{*}{$\begin{array}{l}\text { Group } \\
\text { Variable }\end{array}$} & \multicolumn{5}{|c|}{ Before treatment $(n=110)$} & \multicolumn{5}{|c|}{ After treatment $(n=110)$} & \multicolumn{6}{|c|}{ Change $(n=110)$} \\
\hline & \multirow[t]{2}{*}{ Mean } & \multirow[t]{2}{*}{$S D^{*}$} & \multirow[t]{2}{*}{ Range } & \multicolumn{2}{|c|}{$95 \% \mathrm{Cl} * *$} & \multirow[t]{2}{*}{ Mean } & \multirow[t]{2}{*}{$S D^{*}$} & \multirow[t]{2}{*}{ Range } & \multicolumn{2}{|c|}{$95 \% \mathrm{Cl}^{\star *}$} & \multirow[t]{2}{*}{ Mean } & \multirow[t]{2}{*}{$S D^{*}$} & \multirow[t]{2}{*}{ Range } & \multicolumn{2}{|c|}{$95 \% \mathrm{Cl}^{\star *}$} & \multirow{2}{*}{$\begin{array}{l}\text { Effec } \\
\text { sizes }\end{array}$} \\
\hline & & & & $\begin{array}{l}\text { Lower } \\
\text { limit }\end{array}$ & $\begin{array}{l}\text { Upper } \\
\text { limit }\end{array}$ & & & & $\begin{array}{l}\text { Lower } \\
\text { limit }\end{array}$ & $\begin{array}{l}\text { Upper } \\
\text { limit }\end{array}$ & & & & $\begin{array}{l}\text { Lower } \\
\text { limit }\end{array}$ & $\begin{array}{l}\text { Upper } \\
\text { limit }\end{array}$ & \\
\hline $\begin{array}{l}\text { CPQ*** } \\
\text { Total }(n= \\
68)\end{array}$ & 13.4 & 7.6 & $4-36$ & 11.7 & 15.2 & 9.8 & 7.4 & $0-45$ & 8.1 & 11.6 & 3.9 & 8 & $\begin{array}{l}-15- \\
27\end{array}$ & 2 & 5.8 & 0.49 \\
\hline $\begin{array}{l}\text { Oral } \\
\text { Symptoms }\end{array}$ & 4.2 & 2.3 & $0-10$ & 3.7 & 4.7 & 3 & 2.1 & $0-8$ & 2.5 & 3.5 & 1.4 & 2.9 & $-5-8$ & 0.7 & 2 & 0.48 \\
\hline $\begin{array}{l}\text { Functional } \\
\text { Limitation }\end{array}$ & 2.9 & 2.7 & $0-11$ & 2.3 & 3.5 & 2.5 & 2.2 & $0-9$ & 2 & 3 & 0.5 & 3.2 & $-9-10$ & -0.2 & 1.3 & 0.16 \\
\hline $\begin{array}{l}\text { Emotional } \\
\text { Well-being }\end{array}$ & 3.6 & 3.6 & $0-16$ & 2.8 & 4.4 & 2.1 & 2.7 & $0-13$ & 1.5 & 2.7 & 1.6 & 3.3 & $-8-13$ & 0.8 & 2.4 & 0.48 \\
\hline $\begin{array}{l}\text { Social } \\
\text { Well-being }\end{array}$ & 2.7 & 2.7 & $0-11$ & 2.1 & 3.3 & 2.2 & 2.3 & $0-15$ & 1.7 & 2.7 & 0.4 & 2.8 & $-8-8$ & -0.3 & 1.1 & 0.14 \\
\hline $\begin{array}{l}\text { PIDAQ } \\
\star \star \star \star * \text { Total } \\
(n=42)\end{array}$ & 65.5 & 19.8 & $\begin{array}{l}34- \\
107\end{array}$ & 59.6 & 71.4 & 30.2 & 11.2 & $\begin{array}{l}21- \\
68\end{array}$ & 27.1 & 33.3 & 34.2 & 18.5 & $\begin{array}{l}-16- \\
72\end{array}$ & 28.7 & 39.8 & 1.86 \\
\hline
\end{tabular}

*SD indicates standard deviation, ${ }^{* *}$ confidence interval (Cl);

${ }^{\star * \star} \mathrm{CPQ}$ indicates Child Perceptions Questionnaire, and ****Psychosocial Impact of Dental Aesthetics Questionnaire (PIDAQ).

The before treatment PIDAQ mean total score was $65.5(\mathrm{SD}=19.8, \mathrm{Cl} 59.6-71.4)$ and the after treatment mean total score was $30.2(\mathrm{SD}=11.2, \mathrm{Cl} 27.1-33.3)$.

The mean change in the PIDAQ total scores was $34.3(\mathrm{SD}=18.5, \mathrm{Cl} 28.7-39.8)$. The effect size for the before and after treatment PIDAQ data was 1.86 (Table 1). The mean proportional change for CPQ11-14-ISF-16 scores was $16 \%(\mathrm{SD}=0.63, \mathrm{Cl} 0.01-0.31$ ) and the mean proportional change for PIDAQ scores was $50 \%$ (SD $=0.19, \mathrm{Cl} 0.45-0.56$ ) (Table 3). The global scores were examined using frequencies in each response categories (Table 4). One-way ANOVA analysis demonstrated that CPQ11-14-ISF-16 has poor longitudinal construct validity $(p=0.155)$.

Table 3

The Proportional Changes in the Child Perceptions Questionnaire, and Psychosocial Impact of Dental Aesthetics Questionnaire Total Scores.

\begin{tabular}{|c|c|c|c|c|c|}
\hline \multirow[b]{2}{*}{ Measure } & \multicolumn{5}{|c|}{$\mathrm{CPQ}^{*}(n=68)$ and PIDAQ** $(n=42)$ proportional change } \\
\hline & Mean & $S D^{\star \star \star}$ & Range & $95 \% \mathrm{Cl}{ }^{\star \star \star \star}$ & \\
\hline & & & & Lower limit & Upper limit \\
\hline $\mathrm{CPQ} *$ & $16 \%$ & 0.63 & $-2.5-6$ & 0.01 & 0.31 \\
\hline PIDAQ** & $50 \%$ & 0.19 & $-0.31-0.75$ & 0.45 & 0.56 \\
\hline
\end{tabular}


Table 4

Shows the Data for Total and Domain Child Perceptions Questionnaire Scores Arranged According to the Participants Reported Global Scores

\begin{tabular}{|lcccc|}
\hline \multicolumn{5}{|c|}{ CPQ11-14-ISF-16* Total and Domain scores } \\
\hline & Number of Participants & Mean & $95 \%$ Cl** & \\
\hline Global group & & & Lower limit & Upper limit \\
\hline Greatly improved & 5 & -1.6 & -7.8 & 4.6 \\
\hline Improved & 39 & 4.1 & 1.7 & 6.5 \\
No change & 18 & 6.2 & 1.3 & 11.1 \\
\hline Worse & 6 & 0.2 & -3.7 & 4 \\
\hline *CPQ11-14-ISF-16 indicates Child Perceptions Questionnaire, **Confidence interval (Cl). \\
\hline
\end{tabular}

The IOTN-AC scores from before orthodontic treatment, assessed by the orthodontists indicated that there was a "Moderate need for treatment"; however, the assessments obtained from the laypeople indicated a "Great need for treatment". The mean change in the IOTN-AC scores assessed by the laypeople group was larger than the mean change obtained from the specialists group (Table 5).

Table 5

Summarizes the Variability of the Aesthetic Component Scores and the Aesthetic Changes from Before and After Orthodontic Treatment

\begin{tabular}{|c|c|c|c|c|c|c|c|c|c|c|c|c|}
\hline & \multicolumn{4}{|c|}{ Before treatment IOTN-AC* } & \multicolumn{4}{|c|}{ After treatment IOTN-AC* } & \multicolumn{4}{|c|}{ Changes from treatment IOTN-AC* } \\
\hline & \multirow[t]{2}{*}{ Mean } & \multirow[t]{2}{*}{$S D^{\star \star *}$} & \multicolumn{2}{|c|}{$95 \% \mathrm{Cl}^{\star \star}$} & \multirow[t]{2}{*}{ Mean } & \multirow[t]{2}{*}{$S D^{* * *}$} & \multicolumn{2}{|c|}{$95 \% \mathrm{Cl} *$} & \multirow[t]{2}{*}{ Mean } & \multirow[t]{2}{*}{$S D^{\star \star \star}$} & \multicolumn{2}{|c|}{$95 \% \mathrm{Cl}^{\star \star}$} \\
\hline & & & $\begin{array}{l}\text { Lower } \\
\text { limit }\end{array}$ & $\begin{array}{l}\text { Upper } \\
\text { limit }\end{array}$ & & & $\begin{array}{l}\text { Lower } \\
\text { limit }\end{array}$ & $\begin{array}{l}\text { Upper } \\
\text { limit }\end{array}$ & & & $\begin{array}{l}\text { Lower } \\
\text { limit }\end{array}$ & $\begin{array}{l}\text { Upper } \\
\text { limit }\end{array}$ \\
\hline $\begin{array}{l}\text { Specialists }(n= \\
11)\end{array}$ & 6 & 1.18 & 5.73 & 6.20 & 2 & 0.65 & 1.84 & 2.10 & 4 & 1 & 3.74 & 4.26 \\
\hline $\begin{array}{l}\text { Laypeople }(\mathrm{n}= \\
16)\end{array}$ & 8 & 0.62 & 7.94 & 8.19 & 3.50 & 0.69 & 3.33 & 3.60 & 5.8 & 0.46 & 5.67 & 5.86 \\
\hline
\end{tabular}

The correlation between the change in total CPQ11-14-ISF-16 scores and change in orthodontists' IOTN-AC assessments was small ( $r=-0.084, p=0.516)$. The findings were the same among laypeople IOTN-AC assessments ( $r=-0.140, p=0.225)$. The correlations between the CPQ11-14-ISF-16 subdomain scores, total PIDAQ scores and the IOTN-AC aesthetic changes are shown in Table 6.

Table 6

Associations between changes in the Child perceptions questionnaire subdomains and Psychosocial impact of dental aesthetics questionnaire vs Index of orthodontic treatment need aesthetic component assessments

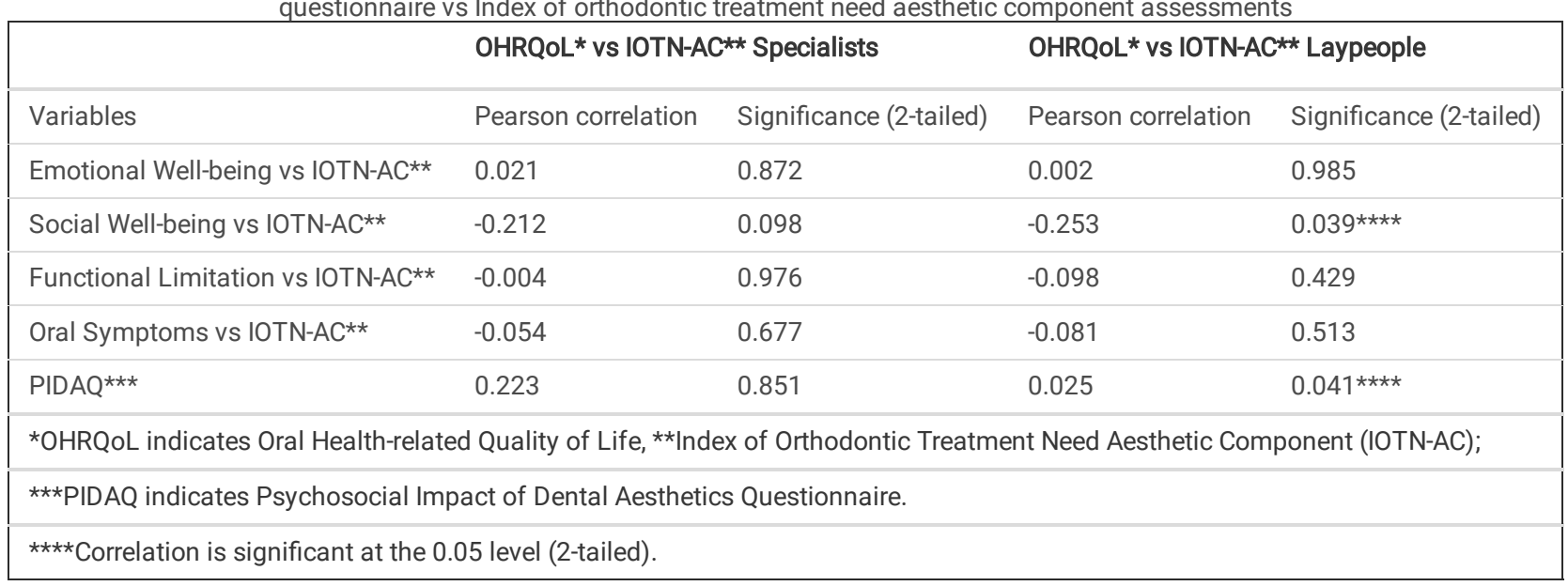

\section{Harms}

There were no adverse events during the study.

\section{Discussion}

In this longitudinal study, we found that the improvements in OHRQoL detected by PIDAQ, were much larger than those detected by CPQ11-14-ISF-16. The correlation between the change in CPQ11-14-ISF-16 scores and change in IOTN-AC assessments, were smaller in comparison to the correlations with PIDAQ. 
However, for the CPQ11-14-ISF-16 subdomains, there were higher, but relatively weak associations between the improvement in the SWB subdomain and improvement in IOTN-AC (Table 6).

In the present study, the improvement in OHRQoL, as shown by CPQ11-14-ISF-16 is considered to be lower than a minimally important difference. ${ }^{17}$ Norman et al., ${ }^{18}$ found that half the standard deviation of baseline scores, represents the most reported minimally important difference. When the CPQ11-14-ISF-16 subdomain scores were taken into consideration, the mean change was highest for the OS and EWB subdomains. When the size and direction of treatment effect in reducing the total CPQ11-14-ISF-16 scores was investigated, this resulted in the effect size to be "medium"; furthermore for the CPQ11-14-ISF-16 FL and SWB subdomains, the effect sizes were"small"; however they were found to be "medium"for both EWB and OS subdomains. These findings are in contrast to the findings of Agou et al. ${ }^{19}$ in a sample of 74 Canadian children, who observed a slightly higher mean change in CPQ11-14-ISF-16 total scores of 4.9 (SD $=14.57$ ) after treatment, this was in comparison to an untreated control group; however when dimensional improvements were accounted for, the changes were higher and significant for the EWB subdomain. The findings of Benson et al. ${ }^{20}$ in the United Kingdom, were almost similar to the current study, they observed a mean change in the total CPQ11-14-ISF-16 scores of $3.2(\mathrm{SD}=6.9 ; p=0.009)$, this was in comparison to a group of participants that had no history of receiving orthodontic treatment and the reported mean change in this group was 2.4 ( $S D=8.8 ; p<0.001)$; however the difference between the two groups was not of statistical significance $(p=0.584)$. It is worth noting that the findings of Benson et al. ${ }^{20}$ were based on a community sample; consequently, such findings are only comparable to this study to a limited extent. On the contrary to these findings, Healey et al. found among a clinical sample of children in New Zealand, that the mean changes in total CPQ11-14-ISF-16 scores after orthodontic treatment (at debond) were small - 0.3 (SD = 14.4) with a "small" effect size of 0; however at the end of their study (mean $=21$ months after treatment) the CPQ11-14-ISF-16 total scores showed significant reduction with a mean change of 10.4 (SD = 7.7) and a "large" effect size of 0.9 ; therefore reporting an overall mean change in CPQ11-14-ISF-16 total scores of 9.8 (SD = 14.6) and a "large" effect size of 0.7 (this was "medium" for all the CPQ11-14-ISF-16 subdomains). ${ }^{21}$ It is not surprising that an improvement in OHRQoL was not detected among their sample immediately following orthodontic treatment, as young people tend to require and need time to adapt to the new arrangement of their teeth.

The "large" improvements in OHRQoL when PIDAQ was used as a measure, are similar to the results of a study conducted on 93 patients in India, where the authors reported "large" (Effect size $=1.47$ ) significant $(P<0.001)$ reductions in mean PIDAQ scores after treatment. ${ }^{22}$ When age was accounted for in relation to the mean change in PIDAQ total scores, a statistical significance $(p=0.04)$ was observed for two age groups (Adolescents $10-19$ years and young adults 20-35 years); however, this was not found to be significant when gender differences (males and females) were considered ( $p=0.31$ ); nonetheless such findings can only be confirmed with larger samples, that would help and be able to account for a variety of individual characteristics. ${ }^{22}$

The discrepancy between examiner IOTN-AC assessments and self-perceived or lay IOTN-AC assessments for the need of orthodontic treatment has been reported in other studies, ${ }^{22-24}$ and this was evident in this study. We found that the aesthetic changes detected by laypeople and orthodontists were variable and the improvements detected in the aesthetic appearance by the laypeople group were slightly larger (Table 5).

The results indicated that the correlation between the change in Total CPQ11-14-ISF-16 scores and change in laypeople and orthodontists IOTN-AC scores were small and statistically non-significant. However, when the CPQ11-14-ISF-16 subdomains were accounted for (Table 6), there were better associations for the SWB subdomain when assessed by both groups; however they were only statistically significant when assessed by the laypeople group. In one study, although total CPQ11-14-ISF-16 scores were associated with the IOTN-AC scores, these changes were mainly influenced by the EWB and SWB subdomains. ${ }^{25}$ This demonstrates the detrimental effects of dental aesthetics on the SWB and EWB of children.

The correlations between the changes in OHRQoL measured by PIDAQ and the IOTN-AC aesthetic changes, were higher and significant in comparison to the associations with CPQ11-14-ISF-16 (Table 6). The findings of the present study are consistent with the findings of Garg et al. ${ }^{22}$ who found statistically significant associations $(\mathrm{P}<0.001)$ between the PIDAQ changes and self-perceived IOTN-AC changes after treatment among their sample. Overall, the findings direct research to employ more condition-specific measures in evaluating patient-reported outcomes in orthodontic treatment, and they also support the perception that orthodontic treatment and improved dental aesthetics have a positive impact on individuals OHRQoL.

Considering the limitations of this study is deemed appropriate at this stage. Lacking an untreated control group among this prospective cohort is considered to be a limitation, as one large community-based longitudinal study among school children, found improvements in OHRQoL (CPQ11-14-ISF-16) among those children with no history of receiving orthodontic treatment i.e. in the control group; ${ }^{19}$ however It could be argued that such changes in OHRQoL among younger age groups are due to natural fluctuations in OHRQoL. Another important weakness of our study is that we did not explore other likely influences on OHRQoL such as age and gender that could have resulted in the observed improvements in CPQ11-14-ISF-16 and PIDAQ scores. The small sample of this study was based on a clinical sample rather than a population based sample; therefore the introduction of sampling bias can be considered a limitation.

The main study's strengths include recruitment of patients in both specialist orthodontic practices and teaching hospitals settings where most patients in the United Kingdom receive treatment, and this implies that this was a "real world" study. Other strengths include that patients were followed up to 3 months after treatment. This is one of the few longitudinal studies assessing OHRQoL among children and young adults using both a generic measure and a conditionspecific measure. Also, alongside the OHRQoL measures, the inclusion of IOTN-AC allowed for the associations between dental aesthetics and OHRQoL to be examined. Whilst the longitudinal construct validity of the CPQ11-14-ISF-16 was examined, it was not possible to test the longitudinal construct validity of PIDAQ, because no global questions were included in the measure.

\section{Conclusion}

- CPQ11-14-ISF-16 is more relevant to the EWB and SWB subdomains, and this demonstrates one of the main disadvantages of generic measures, which is having several items that are not relevant to children. 
- Condition-specific and generic measures can capture different outcomes after orthodontic treatment, with PIDAQ appearing more responsive and measuring much more relevant outcomes, such as SWB and EWB.

- Consensus for the agreements between laypeople and clinicians' IOTN-AC assessments remains equivocal.

Additional evaluations in "real world" settings using controlled longitudinal cohorts involving a larger community and clinical-based samples is important to further confirm and explore these findings.

\section{List Of Abbreviations}

- (OHRQoL) Oral health-related quality of life

- (CPQ11-14-ISF-16) Child Perceptions Questionnaire

- (PIDAQ) Psychosocial Impact of Dental Aesthetics Questionnaire

- (IOTN-AC) Index of Orthodontic Treatment Need Aesthetic Component

- (OS) Oral Symptoms

- (FL) Functional Limitation

- (EWB) Emotional Well-being

- (SWB) Social Well-being

\section{Declarations}

\section{Ethics approval and consent to participate}

Ethical approval was obtained 24 October 2007 from National Research Ethics Service Committee of Yorkshire and the Humber (Leeds West) (Ref: 07/H1307/153; October 24, 2007), in the United Kingdom and the Clinical Research Ethics Committee of Cork Teaching Hospitals (reference number ECM5(2)5208; February 6, 2008) in the Republic of Ireland. Also, obtained from the Ethics Research Committee of the School of Clinical Dentistry (Ref:013575; 19/05/2017). Written informed consent was obtained from all participants and their parents and/or guardians. Participants were free to withdraw their consent at any stage.

\section{Consent for publication}

Not applicable.

\section{Availability of data and materials}

The datasets during and/or analyzed during the current study available from the corresponding author on reasonable request. Also, the protocol is available from the corresponding author on request.

\section{Competing interests}

The authors declare that they have no competing interests.

\section{Funding}

Supported by the Sheffield Hospitals' Charitable Trust, United Kingdom (grant number 7864).

\section{Authors' contributions}

All the authors approved the final draft of the manuscript. ME and PB contributed to data analysis and manuscript writing; ME and PB contributed to participants recruitment and data collection. ME and PB contributed to the conceptual framework of the project.

\section{Permission}

The first author is the owner of the web page included as "Figure 1", hence provides permission to place shots of the web page.

\section{Acknowledgments}

We thank all who took part in the study the participants and the assessors.

\section{References}

1. Brook PH, Shaw WC. The development of an index of orthodontic treatment priority. Eur J Orthod. 1989;11(3):309-20.

2. Doğan AA, Sari E, Uskun E, Sağlam AMŞ. Comparison of orthodontic treatment need by professionals and parents with different socio-demographic characteristics. The European Journal of Orthodontics. 2010;32(6):672-6.

3. Sischo L, Broder H. Oral Health-related Quality of Life What, Why, How, and Future Implications. Journal of dental research. 2011;90(11):1264-70. 
4. Dimberg L, Arnrup K, Bondemark L. The impact of malocclusion on the quality of life among children and adolescents: a systematic review of quantitative studies. Eur J Orthod. 2015;37(3):238-47.

5. Javidi $H$, Vettore M, Benson PE. Does orthodontic treatment before the age of 18 years improve oral health-related quality of life? A systematic review and meta-analysis. American Journal of Orthodontics Dentofacial Orthopedics. 2017;151(4):644-55.

6. Marshman Z, Gibson BJ, Benson PE. Is the short-form Child Perceptions Questionnaire meaningful and relevant to children with malocclusion in the UK? Journal of Orthodontics. 2010;37(1):29-36.

7. Albino J, Cunat J, Fox R, Lewis E, Slakter M, Tedesco L. Variables discriminating individuals who seek orthodontic treatment. Journal of Dental Research. 1981;60(9):1661-7.

8. Klages $\mathrm{U}$, Claus N, Wehrbein H, Zentner A. Development of a questionnaire for assessment of the psychosocial impact of dental aesthetics in young adults. Eur J Orthod. 2006;28(2):103-11.

9. Bellot-Arcís C, Almerich-Silla JM. Psychosocial impact of malocclusion in Spanish adolescents. The Korean Journal of Orthodontics. 2013;43(4):193200.

10. Bucci R, Rongo R, Zito E, Galeotti A, Valletta R, D'Antò V. Cross-cultural adaptation and validation of the Italian Psychosocial Impact of Dental Aesthetics Questionnaire (PIDAQ). Quality of Life Research. 2015;24(3):747-52.

11. Twigge E, Roberts RM, Jamieson L, Dreyer CW, Sampson WJ. The psycho-social impact of malocclusions and treatment expectations of adolescent orthodontic patients. European journal of orthodontics. 2015;38(6):593-601.

12. Klages U, Erbe C, Sandru SD, Brüllman D, Wehrbein H. Psychosocial impact of dental aesthetics in adolescence: validity and reliability of a questionnaire across age-groups. Quality of Life Research. 2015;24(2):379-90.

13. Benson PE, Alexander-Abt J, Cotter S, et al. Resin-modified glass ionomer cement vs composite for orthodontic bonding: A multicenter, single-blind, randomized controlled trial. American Journal of Orthodontics Dentofacial Orthopedics. 2019;155(1):10-8.

14. Jokovic A, Locker D, Guyatt G. Short forms of the Child Perceptions Questionnaire for 11-14-year-old children (CPQ11-14): development and initial evaluation. Health Qual Life Outcomes. 2006;4:4.

15. O'brien C, Benson P, Marshman Z. Evaluation of a quality of life measure for children with malocclusion. Journal of Orthodontics. 2007;34(3):185-93.

16. Cohen J. A power primer. Psychological bulletin. 1992;112(1):155.

17. Tsakos G, Allen PF, Steele JG, Locker D. Interpreting oral health-related quality of life data. Community dentistry oral epidemiology. 2012;40(3):193-200.

18. Norman GR, Sloan JA, Wyrwich KW. Interpretation of changes in health-related quality of life: the remarkable universality of half a standard deviation. Medical care. 2003;41(5):582-92.

19. Agou S, Locker D, Muirhead V, Tompson B, Streiner DL. Does psychological well-being influence oral-health-related quality of life reports in children receiving orthodontic treatment? Am J Orthod Dentofacial Orthop. 2011;139(3):369-77.

20. Benson PE, Da'as T, Johal A, et al. Relationships between dental appearance, self-esteem, socio-economic status, and oral health-related quality of life in UK schoolchildren: A 3-year cohort study. European journal of orthodontics. 2014;37(5):481-90.

21. Healey DL, Gauld RD, Thomson WM. Treatment-associated changes in malocclusion and oral health-related quality of life: A 4-year cohort study. American Journal of Orthodontics Dentofacial Orthopedics. 2016;150(5):811-7.

22. Garg K, Tripathi T, Rai P, Sharma N, Kanase A. Prospective Evaluation of Psychosocial Impact after One Year of Orthodontic Treatment Using PIDAQ Adapted for Indian Population. Journal of Clinical Diagnostic Research: JCDR. 2017;11(8):ZC44.

23. Ahmed B, Gilthorpe MS, Bedi R. Agreement between normative and perceived orthodontic need amongst deprived multiethnic school children in London. Clinical orthodontics research. 2001;4(2):65-71.

24. Kok Y, Mageson P, Harradine N, Sprod A. Comparing a quality of life measure and the Aesthetic Component of the Index of Orthodontic Treatment Need (IOTN) in assessing orthodontic treatment need and concern. Journal of orthodontics. 2004;31(4):312-8.

25. O'Brien K, Wright JL, Conboy F, Macfarlane T, Mandall N. The child perception questionnaire is valid for malocclusions in the United Kingdom. American Journal of Orthodontics Dentofacial Orthopedics. 2006;129(4):536-40.

\section{Figures}




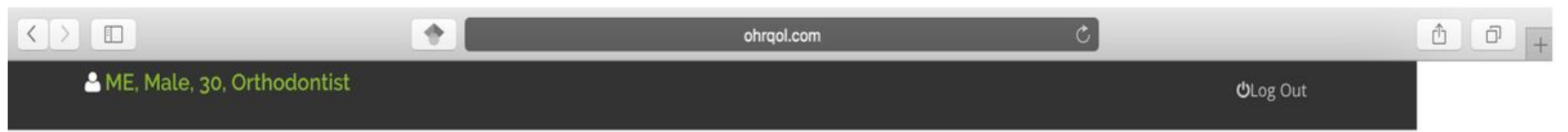

$$
\text { AC - IOTN Score (1-10) }
$$

Please Enter Your Assessment Score Below Each Photo Then Click (Submit Score) Button

\section{Case No.0 Out of 101 Cases}

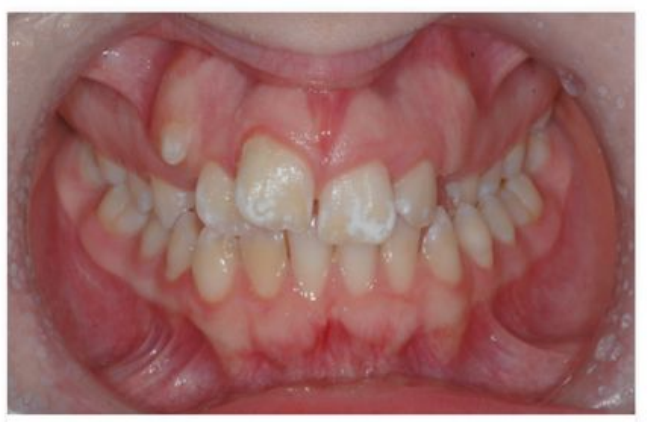

Assessment Score (Before):

$1-10$

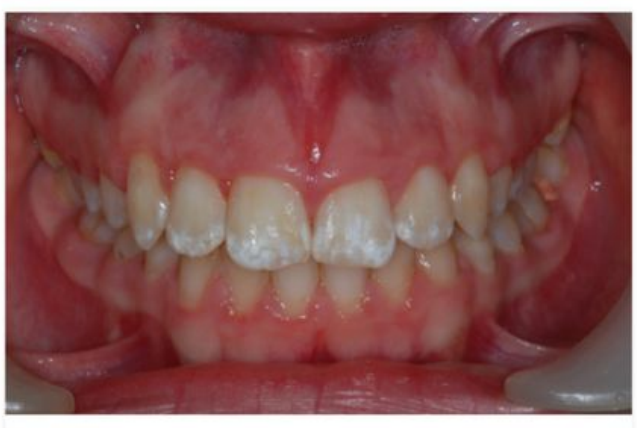

Assessment Score (After):

$1-10$
AC - IOTN Chart

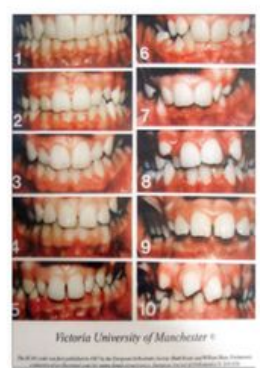

\section{Figure 1}

Online survey webpage. 

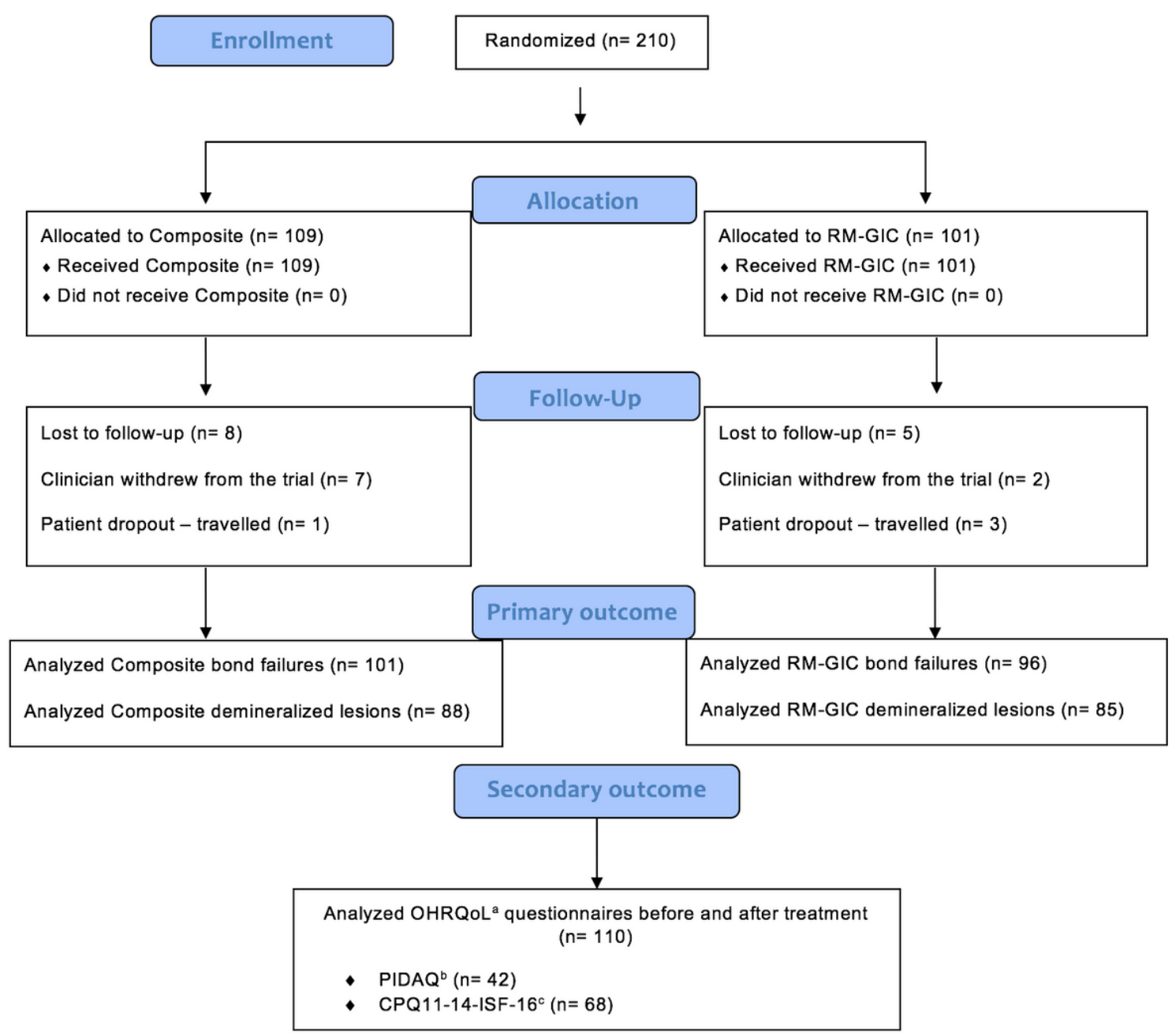

Key 'Oral health-related quality of life; ${ }^{b}$ Psychosocial Impact of Dental Aesthetics Questionnaire; ' Child Perceptions Questionnaire

\section{Figure 2}

Flow of participants through the study

\section{Supplementary Files}

This is a list of supplementary files associated with this preprint. Click to download.

- CONSORT2010Checklist.doc 